\title{
Hubbard model on d-dimensional hypercubes: Exact solution for the two-electron case
}

\author{
Michel Caffarel ${ }^{1}$ and Rémy Mosseri ${ }^{2}$ \\ ${ }^{1}$ CNRS-Laboratoire de Chimie Théorique Tour 22-23, Université Pierre et Marie Curie 4 place Jussieu \\ 75252 Paris Cedex 05; France e-mail: mc@lct.jusssieu.fr \\ ${ }^{2}$ CNRS-Groupe de Physique des Solides Université Paris 6 et Paris 7, 2 place Jussieu; 75251 Paris Cedex 05; France e-mail: \\ mosseri@gps.jussieu.fr
}

(December 1997)

\begin{abstract}
The Hubbard model is exactly solved for two particles with opposite spins on d-dimensional hypercubes. It is shown that the spectrum can be separated into two parts: a trivial (U-independent) part resulting from symmetries of hypercubes and a non-trivial part expressed as a single-impurity problem on a set of finite chains of size $d^{\prime}+1\left(d^{\prime} \leq d\right)$. The exact expression for the one-particle Green's function is given. Finally, we discuss the extension of these results to standard hypercubic lattices with periodic boundary conditions.
\end{abstract}

PACS No: $71.10+\mathrm{x} ; 71.27+\mathrm{a}$

One of the most widely used models to describe strongly correlated fermions systems is the Hubbard model and its various extensions. Unfortunately, only limited exact information about its physical properties is available. For the one-dimensional lattice the celebrated solution of Lieb and $\mathrm{Wu}$ [1] provides the exact eigenspectrum of the model. In the opposite limit of large dimensions investigated very recently [2], some almost exact results have also been obtained. However, for intermediate dimensions, and particularly for the very important two-dimensional case believed to be relevant to high- $\mathrm{T}_{c}$ superconductivity, very little is at our disposal. Most of the results reported so far have been obtained either from numerical solutions on very small clusters (subject to important finite-size effects) or by using a variety of approximate analytical methods (with domains of validity and/or systematic errors difficult to evaluate) [3].

In this paper we present an exact solution for the Hubbard model with first-neighbor hopping term $t$ and onsite interaction energy $U$

$$
H=-t \sum_{<i j>\sigma} c_{i \sigma}^{+} c_{j \sigma}+U \sum_{i} c_{i \uparrow}^{+} c_{i \uparrow} c_{i \downarrow}^{+} c_{i \downarrow}=T(t)+V(U),
$$

for two particles with opposite spins on a d-dimensional hypercube $\gamma_{d}$. The hypercube $\gamma_{d}$ is defined as the set of $N=2^{d}$ sites whose $d$ coordinates are either 0 or 1 . Although our solution is yet limited to the two-electron case, to exhibit exact results for a truly interacting system in dimension greater than one is clearly of primary interest. This is particularly true since hypercubes are related to usual cubic lattices with periodic boundary conditions: $\gamma_{2 d}$ is topologically equivalent to a d-dimensional hypercubic lattice of linear size equal to 4 with periodic boundary conditions, noted $Z_{4}^{d}$ (i.e., $\gamma_{2}$ is equivalent to the 4 -sites ring, $\gamma_{4}$ equivalent to the 2 -dimensional $4 \times 4$ cluster with periodic conditions [4,5], etc..), while $\gamma_{2 d+1}$ is equivalent to two translated linked copies of $Z_{4}^{d}$. To solve the above Hamiltonian we first use an abelian subgroup of the full $\gamma_{d}$ point group, to block-diagonalize the initial matrix of size $2^{2 d}$ into $2^{d}$ blocks of size $2^{d}$. Quite interestingly, these smaller submatrices correspond to a simple family of effective one-electron Hamiltonians defined on $\gamma_{d^{\prime}}\left(d^{\prime} \leq d\right)$, some of which having a single-impurity site. In a second step, we show how to further reduce these matrices into smaller blocks of size $d^{\prime}+1$ (instead of $2^{d}$ ), corresponding to finite chains of size $d^{\prime}+1$ with one impurity site at one end and new specific hopping terms. Using a standard approach for impurity problems we provide a closed expression sum rule for the eigenspectrum. We also derive the exact expression for the one-particle Green's function. Finally, the extension of these results to hypercubic lattices with periodic boundary conditions is briefly discussed.

Let us define $\Lambda_{d}$, an abelian subgroup of the full $\gamma_{d}$ point group, generated by the $d$ reflections into perpendicular $(d-1)$-dimensional planes meeting at the $\gamma_{d}$ centre,

$$
\Lambda_{d}=\left\{\pi_{i}, i=1 \ldots d \mid \pi_{i}^{2}=E, \pi_{i} \pi_{j}=\pi_{j} \pi_{i}\right\}
$$

where $E$ is the identity operator. It is easy to show that $\Lambda_{d}$ has $2^{d}$ elements (owing to the commutativity between the mirror operations), and that the orbit of a generic point under $\Lambda_{d}$ is a hypercube $\gamma_{d}$. Let us number the $\Lambda_{d}$ elements $L$ by integers between 0 and $2^{d}-1$, in the following way: $L$ being a product of mirrors $\pi_{i}$, let $L$ be the number, written in base-two, whose corresponding $i^{t h}$ digits are equal to one $\left(\pi_{1}\right.$ is $L=1, \pi_{1} \pi_{2} \pi_{3}$ is $L=7$, etc...). Let us also locate the sites according to their numbering in base-two: The $\mathrm{i}^{\text {th }}$ coordinate is equal to the $\mathrm{i}^{t h}$ digit. Now, the action of the symmetry operation $L$, onto a site $s$, noted as the "special product" $L \bullet s$, can be explicited: each time a digit equals 1 in $L$, it switches the corresponding digit in $s$. For example, in 3 dimensions, $7 \bullet 1=6$, with corresponds in coordinates to $\pi_{1} \pi_{2} \pi_{3}(0,0,1)=(1,1,0)$. To construct the irreducible representations (irreps) and the character table of $\Lambda_{d}$, it is useful to remark that this group is isomorphic to $d$ times the tensorial product with itself of the 
two-element group $Z_{2}=\left\{E, \pi_{1}\right\}=\Lambda_{1}[6]$. This is nothing else than the group theory counterpart of the natural construction of $\gamma_{d}$ as two displaced copies of $\gamma_{d-1}$. The $\Lambda_{d}$ irreps are easily obtained as tensor products of the two irreps $\Gamma_{0}$ and $\Gamma_{1}$ of $Z_{2}$. As an illustration, the character table fo $\Lambda_{2}=Z_{2} \times Z_{2}$ is shown in Table II. All the irreps are uni-dimensional $\left(\Lambda_{d}\right.$ is abelian), and the character table is identical to standard Hadamard matrices [7], which are orthogonal matrices with elements \pm 1 (note also that lines and columns of Hadamard matrices represent the non-interacting one-electron orbitals of the problem). Note that the $N\left(=2^{d}\right)$ irreps can also be labelled by integers $M$, through their base-two decomposition, by ordering the different occurences of $Z_{2}$ in the tensorial product, and fixing the corresponding digit to 0 or 1 , whether the irreps $\Gamma_{0}$ and $\Gamma_{1}$ occur.

TABLE I. Character table for $\Lambda_{2}$. The irreducible representations are numbered by an integer $M$ as defined in text.

\begin{tabular}{cccrrr}
\hline \hline $\mathrm{M}$ & & $\mathrm{E}$ & $\pi_{1}$ & $\pi_{2}$ & $\pi_{1} \pi_{2}$ \\
\hline 0 & $\Gamma_{0} \times \Gamma_{0}$ & 1 & 1 & 1 & 1 \\
1 & $\Gamma_{1} \times \Gamma_{0}$ & 1 & -1 & 1 & -1 \\
2 & $\Gamma_{0} \times \Gamma_{1}$ & 1 & 1 & -1 & -1 \\
3 & $\Gamma_{1} \times \Gamma_{1}$ & 1 & -1 & -1 & 1 \\
\hline \hline
\end{tabular}

Let us consider first the one-electron tight-binding spectrum with hopping term $t$. Each eigenstate belongs to a given irrep of $\Lambda_{d}$, and reads

$\left|M>=\frac{1}{\sqrt{2^{d}}} \sum_{R \in \Lambda_{d}} \chi_{R}^{M} R(\mid 0>)=\frac{1}{\sqrt{2^{d}}} \sum_{r=0}^{N-1}(-1)^{m_{M, r}}\right| r \bullet 0>$

where $m_{M, r}$ is the number of digits equal to 1 simultaneously in $M$ and $r$ (we have used the above defined special product $\bullet$ to denote the action of the symmetry operation $R$ onto the basis kets). Since all the sites are equivalent, it is sufficient to consider the interaction of the site number 0 with its $d$ first neighbors which have exactly one digit equal to 1 and belong to the set $V_{d}=\left\{v_{j}=2^{j}, j=0 \ldots d-1\right\}$. As a result, one finds that the eigenenergies read

$$
E_{M}=\sum_{j=0}^{d-1} \chi_{v_{j}}^{M}=-t\left(d-2 N_{M}\right)
$$

where $N_{M}$ is the number of digits equal to 1 in $M$. Since $M$ runs from 0 to $2^{d}-1$, the $\gamma_{d}$ spectrum consists of $d+1$ levels, from $-d t$ to $d t$, with equal spacing $2 t$. The degeneracy of a $E_{M}$ level is the standard binomial coefficient counting the number of sets of $N_{M}$ digits among $d$. As $d$ tends to infinity, one recovers a gaussian-shaped spectrum, as for the infinite-dimensional cubic lattice.

Let us now consider the two-electron case (electrons with opposite spins). The full Hamiltonian matrix, of size $2^{2 d}$, is readily block-diagonalized into $2^{d}$ blocks of size $2^{d}$, associated with each representation $M$. For a given $M$, one constructs a basis with $2^{d}$ kets $|M, l\rangle$

$$
\begin{aligned}
& \mid M, l>=\frac{1}{\sqrt{2^{d}}} \sum_{R \in \Lambda_{d}} \chi_{R}^{M} R(\mid 0 \uparrow, l \downarrow>) \\
& =\frac{1}{\sqrt{2^{d}}} \sum_{r=0}^{N-1} \chi_{r}^{M} \mid(r \bullet 0) \uparrow,(r \bullet l) \downarrow>
\end{aligned}
$$

It is clear that $U$ only occurs in $<M, 0|V(U)| M, 0>$, while the kinetic part reads

$$
\begin{gathered}
<M, l|T| M, l^{\prime}>= \\
\frac{1}{2^{d}} \sum_{r=0}^{N-1} \sum_{r^{\prime}=0}^{N-1} \chi_{r}^{M} \chi_{r^{\prime}}^{M}<(r \bullet 0) \uparrow(r \bullet l) \downarrow|T|\left(r^{\prime} \bullet 0\right) \uparrow,\left(r^{\prime} \bullet l^{\prime}\right) \downarrow> \\
=\frac{1}{2^{d}} \sum_{r=0}^{N-1}\left[\chi_{r}^{M} \chi_{r}^{M}<(r \bullet 0) \uparrow(r \bullet l) \downarrow|T|(r \bullet 0) \uparrow,\left(r \bullet l^{\prime}\right) \downarrow>\right. \\
+\sum_{j=0}^{d-1} \chi_{r}^{M} \chi_{v_{j}}^{M} \bullet r
\end{gathered}
$$




$$
\left.<(r \bullet 0) \uparrow(r \bullet l) \downarrow|T|\left(v_{j} \bullet r \bullet 0\right) \uparrow,\left(v_{j} \bullet r \bullet l^{\prime}\right) \downarrow>\right]
$$

In the latter expression, we have decoupled the terms where the $\downarrow$ spin jumps (first part) from those where the $\uparrow$ spin jumps. In the latter part (sum over $j$ ) the only nonvanishing term corresponds to $r \bullet l=v_{j} \bullet r \bullet l^{\prime} \rightarrow$ $l^{\prime}=v_{j} \bullet l$, which means $l$ and $l^{\prime}$ neighbors (recall that the group is abelian and its elements are their own inverse). One finally gets that the nonvanishing kinetic terms read

$$
<M, l|T| M, v_{j} \bullet l>=-\frac{t}{2^{d}} \sum_{r=0}^{N-1}\left(1+\chi_{r}^{M} \chi_{v_{j}}^{M} \bullet r\right)
$$

The block matrix associated to the irrep $M$ corresponds therefore to an effective one-electron tightbinding Hamiltonian, with sites labelled by $l$, kets $\mid M, l>$ and hopping terms given by the above expression (7). A simple case is provided by the identity irrep $M=0$, for which $\chi_{r}^{M}=1$ for any $r$. The effective Hamiltonian $H^{(0)}$ corresponds to a hypercube $\gamma_{d}$, with one "impurity" site (with diagonal term $U$ ) and a constant hopping term $-2 t$. We now show that the other irreps correspond to effective Hamiltonian $H^{(M)}$ on hypercubes $\gamma_{d^{\prime}}$ with $d^{\prime}<d$. One has to evaluate the right-hand part of equation (7). By definition of the $v_{j}$ (mirrors which connect a site to one of its first neighbors), $r$ and $v_{j} \bullet r$ differ by one digit, which depends on $j$, not on $r$. If this digit takes the value 1 in the base-two decomposition of $M$, then $\chi_{r}^{M} \chi_{v_{j}}^{M} \bullet r=-1$ for any $r$, and the corresponding $H^{(M)}$ matrix element vanishes. $H^{(M)}$ therefore corresponds to a $\gamma_{d}$ in which families of parallel edges have been cut (those orthogonal to the mirrors labelled by the digits 1 in the decomposition of $M$ ). Figure 1 displays graphically this general result for $d=2$ and $d=3$.

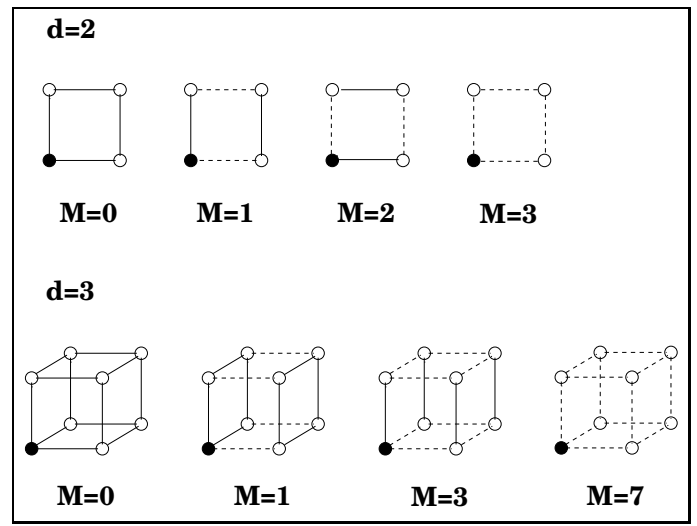

Fig. 1. Graphical illustration of the effective Hamiltonian $H^{(M)}$ associated with the irrep $M$. Full circles correspond to impurity sites with a diagonal term $U$, full edges to hopping terms equal to $-2 t$, and dotted lines to the edges which are cut in the given irrep.

a) $d=2$, the four irreps, corresponding to one square with one impurity, two times two segments, one containing an impurity site, and finally four isolated sites (one being an impurity with a diagonal term $U$ ).

b) the case $d=3$. Four irreps among the eight are shown.
So, at this step, the spectrum is solved in terms of one-electron tight-binding models on hypercubes of dimension lower or equal to $d$. The only non-trivial part corresponds to those part, in the hypercube decomposition, which contain the impurity site [8]. We now show how to further greatly reduce their complexity.

To do that it is convenient to re-express the effective Hamiltonian $H$ (hereafter we will drop the superscript (0) since only the $M=0$ case has finally to be considered) in the one-electron basis, Eq.(33). It is easy to verify that $H$ can be written in the form

$$
H=\operatorname{Diag}(\epsilon)+\frac{U}{N} I_{N}
$$

where $\operatorname{Diag}(\epsilon)$ is a diagonal matrix whose entries are the $2^{d}$ free electron-solutions of the hypercube with hopping $2 t$ (solution at $U=0$ ) and $I_{N}$ is the $N \times N$ matrix with unit entries for all $i$ and $j$. The latter part is nothing but a projection operator on the eigenstate $\mid v_{0}>$ with energy $U$ and $H$ can be rewritten

$$
H=\operatorname{Diag}(\epsilon)+U\left|v_{0}><v_{0}\right|
$$

with $\left(v_{0}\right)_{i}=\frac{1}{\sqrt{N}} i=1, \ldots, N$.

To proceed further we define for each subspace corresponding to a given value $\epsilon_{i}(d+1$ subspaces of degeneracy $g_{i}$, the binomial coefficient) a basis consisting of the normalized vectors $v_{1}^{(i)}=\frac{1}{\sqrt{g_{i}}}(1, \ldots, 1)$ and a set of vectors $\left\{v_{k}^{(i)}, k \neq 1\right\}$ spanning the subspace orthogonal to $v_{1}^{(i)}$. In this new basis only the $(d+1)$ vectors $v_{1}$ have a non-zero overlap with $\left|v_{0}\right\rangle$, and $H$, of dimension $2^{d}$, decomposes into a diagonal matrix having $2^{d}-(d+1)$ trivial solutions $\epsilon_{i}$, and a residual U-dependent part, noted $\mathcal{H}$, given by the matrix of linear size $(d+1)$ written in the form

$$
\mathcal{H}=\operatorname{Diag}(\epsilon)+U|v><v| \equiv \mathcal{H}_{0}+\mathcal{V}
$$

where $\mid v>$ has components $v_{i}=\sqrt{g_{i} / N}$ and the $\epsilon$ 's represent now the $(\mathrm{d}+1)$ distinct free-electron energies:

$$
\epsilon_{i}=-2 t(d-2 i) \quad i=0, \ldots, d .
$$

An alternative representation consists in going back to the basis where the potential operator $\mathcal{V}$ is diagonal. $H$ is then found to be tridiagonal (one-dimensional tightbinding model) with off-diagonal hopping term given by $t_{i, i+1}=2 t \sqrt{(d-i)(i+1)}, \quad i=0$ to $d-1$ and a diagonal $U$-contribution at the initial site (here numbered $0)$. In other words, the problem is mapped onto a singleimpurity problem on a finite chain of linear size $(d+1)$. Figure 2 illustrates this result for $d \leq 3$.

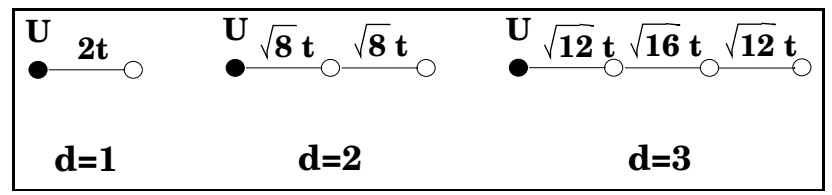


Fig. 2. Graphical illustration of the single-impurity oneelectron problems associated with the non-trivial part of the spectrum (general formula given in text).

It is known that eigenspectrum of single-impurity problems can be expressed under the form of a sum rule expression using the Koster-Slater approach [9]. This result can be briefly re-obtained as follows. First, the following operator identity 10 is invoked

$$
\begin{gathered}
(\mathcal{H}-z)^{-1}=\left(\mathcal{H}_{0}-z\right)^{-1} \\
-\frac{U}{1+U G_{v v}^{(0)}(z)} \frac{1}{\left(\mathcal{H}_{0}-z\right)}|v><v| \frac{1}{\left(\mathcal{H}_{0}-z\right)}
\end{gathered}
$$

where $z$ is an arbitrary complex number and $G_{v v}^{(0)}$ the unperturbed Green's function given by $G_{v v}^{(0)}(z)=<$ $v\left|\left(\mathcal{H}_{0}-z\right)^{-1}\right| v>$. Then, using this identity the fully interacting Green's function, $G_{v v}(z)$, can be written as

$$
G_{v v}(z)=\frac{G_{v v}^{(0)}(z)}{1+U G_{v v}^{(0)}(z)}
$$

Searching for poles of $G_{v v}(z)$ we get

$$
\frac{1}{N} \sum_{i=0}^{d} \frac{g_{i}}{E-\epsilon_{i}}=\frac{1}{U}
$$

Eq.(14) is the final closed expression determining the non-trivial part of the spectrum. It is easily shown that this equation admits $(d+1)$ distinct solutions we shall denote as $\operatorname{Spect}^{(d)}(U)$.

To summarize, the set of $2^{2 d}$ eigenvalues of the hypercube $\gamma_{d}$ consists of a U-dependent part given by the collection of the non-trivial spectra at dimensions lower or equal to $d$ :

$$
\bigoplus_{i=0}^{d}\left(\begin{array}{c}
d \\
i
\end{array}\right) \operatorname{Spect}^{(i)}(U)
$$

(note that here the binomial coefficient counts the number of occurences of $\gamma_{i}$ hypercubes with an impurity site in the decomposition of the initial $\gamma_{d}$, see Fig.11) and a trivial U-independent part given by:

$$
E_{l}=2 t l \quad l=-d \ldots d
$$

with degeneracy:

$$
g_{l}=\sum_{i=|l|}^{\prime d}\left[2^{d-i}\left(\begin{array}{c}
i \\
\frac{l+i}{2}
\end{array}\right)-1\right]\left(\begin{array}{c}
d \\
i
\end{array}\right)
$$

the prime in $\sum^{\prime}$ indicating that the summation over $i$ is done with an increment of two. Formula (17) can be obtained by tracing back the contributions due to the different irreps and those issued from the internal symmetry of hypercubes (trivial solutions extracted when passing from Eq.(9)) to Eq.(10)). The ground-state energy is not degenerate and corresponds to the lowest solution in $\operatorname{Spect}^{(d)}(U)$. Note also that in the limit of large dimensions, and after proper renormalization of the parameters of the Hamiltonian, an infinite-dimensional model can be defined, as done recently for a number of correlated fermions models, see [2].

Let us now consider dynamical properties. We are interested in evaluating the one-particle Green's function defined by

$$
G_{k}^{(d)}(z, U) \equiv<\psi_{0}\left|a_{k} \frac{1}{H-z} a_{k}^{+}\right| \psi_{0}>
$$

where $\left|\psi_{0}\right\rangle$ denotes the one-particle ground-state consisting of one electron of given spin and $a_{k}^{+}$creates one electron of opposite spin in a one-particle state, noted $k$. Hereafter, $k$ varies from 0 to $d$ and labels one of the $(d+1)$ degenerate subspaces of the one-electron problem, the ground-state corresponding to $k=0$. Now, remark that vector $a_{k}^{+} \mid \psi_{0}>$ belongs to the subspace corresponding to the decomposition on hypercubes of dimension $(d-k)$. There exist $2^{k}$ different families of hypercubes having such a dimension (See, fig. 11) among which only one has the U-impurity site. Accordingly, we get the following result

$G_{k}^{(d)}(z, U)=\frac{1}{2^{k}} G_{0}^{(d-k)}(z, U)+\left(1-\frac{1}{2^{k}}\right) G_{0}^{(d-k)}(z, U=0)$.

Now, we need to evaluate the fundamental quantity $G_{0}^{\left(d^{\prime}\right)}(z, U)$ with Hamiltonian (10), the dimension $d^{\prime}$ ranging from 0 to $d$. For that we note that the ket $\mid 0>\equiv$ $a_{0}^{+} \mid \psi_{0}>$ represents the (first) basis element corresponding to the diagonal energy $\epsilon_{0}$ in representation (10). Projecting out identity (12) onto vector $\mid 0>$ and expressing the different quantities in terms of the non-interacting spectrum we get:

$$
\begin{gathered}
G_{0}^{\left(d^{\prime}\right)}(z, U)= \\
\left.\frac{1}{\epsilon_{0}^{\left(d^{\prime}\right)}-z}-\frac{U}{2^{d^{\prime}}\left(\epsilon_{0}^{\left(d^{\prime}\right)}-z\right)^{2}\left(1+\frac{U}{2^{d^{\prime}}} \sum_{i=0}^{d^{\prime}} \frac{g_{i}}{\epsilon_{i}\left(d^{\prime}\right)}-z\right.}\right)
\end{gathered}
$$

This equation together with Eqs.(11) and (19) give the exact one-particle Green's function of the problem.

Generalization of this approach to higher fillings is presently under investigation. We have already found that the existence of large fractions of U-independent eigenvalues is still valid for some specific fillings. However, the underlying structure for the U-dependent part of the spectrum is more difficult to elucidate.

Back to the two-electron case, we would like to mention that, by using rotations instead of reflections, a similar calculation can be done for standard d-dimensional hypercubic lattices of linear size $\mathrm{L}$ and periodic boundary conditions $\left(Z_{L}^{d}\right)$. We have found that the problem 
can still be mapped onto a family of $L^{d}$ single-impurity one-electron effective problems defined on the very same lattice, $Z_{L}^{d}$. Each irrep corresponds to a value of a ddimensional integer vector $\vec{M}\left(M_{i}=0 \ldots L-1\right)$, or more physically, to a value for the total momentum $\vec{K}=\frac{2 \pi}{L} \vec{M}$. The only difference between irreps lies in the value of the hopping term which depends explicitly on $\vec{M}$. More precisely, for each irrep labelled by $\vec{K}$, Eq.(14) now reads

$$
\frac{1}{L^{d}} \sum_{n_{1}=0}^{L-1} \ldots \sum_{n_{d}=0}^{L-1} \frac{1}{E-\epsilon_{\vec{n}}^{(\vec{K})}}=\frac{1}{U}
$$

where the non-interacting energies $\epsilon^{(\vec{K})}$ are given by

$$
\epsilon_{\vec{n}}^{(\vec{K})}=-4 t \sum_{i=1}^{d} \cos \left(K_{i} / 2\right) \cos \left(k_{i}+K_{i} / 2\right)
$$

with $\vec{K}=\frac{2 \pi}{L} \vec{M}$ and $\vec{k}=\frac{2 \pi}{L} \vec{n}$. In contrast with hypercubes, the sum in Eq.(21) runs over $L^{d}$ values and cannot be further reduced. Note that, since the effective hopping term varies with the irrep (and can even vanish), both localized and resonant states (irreps by irreps) may be present. Finally, it is worth noticing that in the $d=1$ case the Bethe ansatz equations for two particles [1]:

$$
\tan \frac{k_{1} L}{2}=\frac{U}{2 t\left(\sin k_{1}-\sin k_{2}\right)}
$$

with $E=-2 t\left(\cos k_{1}+\cos k_{2}\right)$ and $k_{1}+k_{2}=\frac{2 \pi}{L} M(M=$ $0 \ldots L-1)$ can be recovered from Eq.(21) after simple but tedious algebra.

This work was supported by the "Centre National de la Recherche Scientifique" (C.N.R.S.)

[1] E.H. Lieb and F.Y. Wu, Phys. Rev. Lett 20, 1445 (1968).

[2] See, e.g., A. Georges, G. Kotliar, W. Krauth, and M.J. Rozenberg, Rev. Mod. Phys. 68, 13 (1996).

[3] See, e.g., E. Dagotto, Rev. Mod. Phys. 66, 763 (1994).

[4] R. Mosseri, D.P. DiVincenzo, J.F. Sadoc, and M.H. Brodsky, Phys. Rev. B 32, 3974 (1985).

[5] H. Bruus and J.C. Anglès d'Auriac, Phys. Rev. B 55, 9142 (1997).

[6] For $d=4, \Lambda_{4}$ coincides with the group $\mathcal{A}$ used in ref. [(Appendix 1) where the full $\gamma_{4}$ symmetry group is used to diagonalize the Hamiltonian.

[7] M. Hall, Jr. Combinatorial Theory edited by WileyInterscience, Series in Discrete Mathematics (1986).

[8] The occurence of a large fraction of trivial states was noted for $d=2, \| 5$. . Our results put new light on this topic for general $d$.

[9] G.F. Koster and J.C. Slater, Phys. Rev. 95, 1167 (1954).
[10] Identity obtained by making a series expansion of operator $(\mathcal{H}-z)^{-1}$ in terms of $\mathcal{V}$ and using the fact that $\mathcal{V}$ is a projector. 